\title{
«La robe sans couture de la réalité »: André Bazin et l’apologie du réalisme cinématographique
}

\author{
Par OLEG LEBEDEV \\ Université catholique de Louvain
}

Dès le début, le bouleversement des modes de perception amené par la machine à reproduire la vie appelée " le cinématographe » était inséparable des transformations sociales et des changements culturels du début du $\mathrm{XX}^{\mathrm{e}}$ siècle. Avec la vitesse d'un éclair, une nouvelle distraction nous fait voyager et nous emmène au bout du globe ; sur l'écran filent une automobile, un vélo, des policiers ; un train roule à toute allure sur un opérateur couché au ras des rails ; les ombres animées éveillent des émotions splendides chez les spectateurs fascinés, sans exiger d'eux trop de culture, ni trop d'attention, ni même trop d'effort pour les suivre; très proche de l'art le plus populaire, voire vulgaire, véritable théâtre démocratique du futur, cette ingénieuse invention rend aussi pourtant la vérité simple des beautés délivrées de toute théorie. Pour les artistes, c'était un moyen plastique énergique et enfiévré. Pour les historiens de l'art, l'expression d'une synthèse supérieure des formes artistiques traditionnelles. Pour les scientifiques, la possibilité d'intercepter des mouvements très rapides ou très lents, et de fournir un moyen de démonstration des phénomènes de la nature. Pour les philosophes, l'annonce d'émotions morales inédites et de suggestions de nouvelles métaphysiques. Bref, le cinéma est un art qui pouvait accompagner enfin en rythme un monde luimême en marche, un art mécanique qui pouvait devenir le miroir d'une époque en mutation ${ }^{1}$.

Pourtant, penser le cinéma avec fièvre et précipitation, à l'aube du $\mathrm{XX}^{\mathrm{e}}$ siècle, était alors déjà penser à l'endroit même d'une contradiction, à l'inter-

\footnotetext{
${ }^{1}$ Sur la réception de l'invention et les premiers discours sur le cinéma, $c f$. le recueil de textes : Daniel Banda et José Moure (dir.), Le Cinéma : naissance d'un art, 1895 1920, Paris, Éditions Flammarion, 2008.
} 
section de l'image comprise comme une matrice constituée de millions de pointillés référant à la texture sensible des choses, et l'image conçue comme représentation dotée d'un pouvoir de signification. Une fois l'effervescence passée, ce sont les étranges fiançailles de ces deux principes, du certificat de présence et de la puissance signifiante, de la monstration et de la narration, qui ont donné l'impulsion aux critiques et théoriciens. À ce sujet, le problème de réalité était peut-être plus prompt que nul autre à condenser et à déployer les arguments enchevêtrés mis en jeu par l'exigence que le cinéma soit autre chose qu'une simple frivolité. Ainsi, dès le début, on a loué le cinématographe de donner un monde tel qu'en lui-même, objectivement. Ce qui a fasciné à la naissance du cinéma, c'est donc la force et le mystère de l'image analogique, le petit miracle automatique de l'enregistrement synchrone qui restitue une illusion quasi-parfaite.

Parmi les nombreux théoriciens et critiques, c'est peut-être André Bazin qui a atteint la proximité d'une pensée véritable concernant cette capacité de l'enregistré à rendre l'ambivalence des choses, à les saisir dans leur fugacité. Héritant en droite ligne des premiers discours sur le cinématographe, avec ce que ceux-ci avaient d'oscillant entre enthousiasme et inquiétude, André Bazin fixe dans les années 1940 dans quelques articles théoriques - regroupés dans un recueil portant le titre très platonicien Qu'est-ce que le cinéma? - des idées qui restent aujourd'hui encore des repères majeurs. Grâce à la forme précise de son écriture et de son regard toujours très pénétrant, ce critique de cinéma qui n'a été qu'occasionnellement un philosophe à la recherche de «l'essence», est devenu pour les générations à venir un guetteur, un veilleur pour la réflexion sur l'esthétique filmique, laquelle était pour Bazin inséparable de l'épiphanie du réel rendue possible par la technique objective de reproduction visuelle et sonore.

Mais il y a plusieurs façons de comprendre cette glorification. La première a trait à la prédisposition profonde, structurelle et technique du médium à l'égard d'un réel non médiatisé. En effet, mieux encore que la photographie, le cinématographe hérite de l'enregistrement mécanisé ces deux propriétés que sont la référentialité et l'automatisme. En vertu de l'ontogenèse de ses images, le cinéma propose des vestiges matériels du modèle, il ratifie ce qu'il représente. Par cette puissance constative, l'indifférence scrupuleuse de la machine ne fait rien d'autre que générer patiemment les empreintes de ce monde; ce qui était là, présent en face de la caméra, est nécessairement ce qui sera projeté, donc en droit ce qui pourra être vu n'importe où et partout. Mais tout aussi bien, l'image-copie est produite d'une façon toute particulière : en supprimant l'artiste, en retirant l'agent humain de la reproduction. De ce côté aussi, le cinéma accomplit 
mieux que tout art le lien non médiatisé de l'image au réel, en laissant celuici inaltéré par la main humaine. Dans l'idée de la fenêtre ouverte sur le monde d'Alberti, la surface de la toile était certes considérée comme une matière transparente, mais la peinture avait encore pour fonction de narrer ${ }^{1}$; bref, elle se mouvait dans l'élément de l'historia. Mais au cinéma, la caméra n'intervient pas parmi les choses qu'elle filme, elle laisse simplement le monde être et imprimer ses émanations lumineuses et sonores sur la surface sensible de la pellicule; manière de déléguer à la machine le soin d'une objectivité qui est indifférente à nous. Bazin, en ce sens très proche des discours sur la révolution analogique connus au début du $\mathrm{XX}^{\mathrm{e}}$ siècle, demeura très sensible à cet avènement d'une nouvelle catégorie d'images, qu'il compare à la formation naturelle de cristaux :

Tous les arts sont fondés sur la présence de l'homme; dans la seule photographie nous jouissons de son absence. Elle agit sur nous en tant que phénomène «naturel », comme une fleur de cristal ou de neige dont la beauté est inséparable des origines végétales ou telluriques ${ }^{2}$.

Pourtant cette position sur l'ontogenèse des images et la neutralité intrinsèque du médium filmique, constamment attribuée à Bazin, a tout d'un réalisme franchement naïf, qu'on peut schématiser par un énoncé très simple : le cinéma est la reproduction mécanisée du réel. En tant que tel, cet art dégagerait les structures profondes de l'événement filmé et, dans sa suspension des beautés trop académiques, atteindrait enfin l'autoprésentation d'un monde inerte indifférent à l'humanité. Au lieu d'évoquer et de décrire, il «écrit le réel », " en élevant jusqu'à une authentique signification métaphysique l'effet d'un objectivisme inhumain et minéral $»^{3}$.

Mais que conclure de là, sinon que Bazin est un bourgeois teinté d'idéalisme, un humaniste mystique ? Et chaque année, revenant peu ou prou aux accents religieux de son œuvre, quelqu'un semble redécouvrir qu'assurément, Bazin était trop naïf, qu'eu égard au cinéma il n'y a pas de réalité, pas de fait brut, aucun objectivisme, mais artifice, relativité sémantique et expression humaine - ce pourquoi le cinéma est un art poétique capable de jouer avec le mouvement des formes lumineuses et pas une inscription automatique du réel sur la pellicule. Il est vrai qu'on peut se demander légi-

${ }^{1}$ Jean-Louis Comolli, Cinéma contre spectacle, Lagrasse, Éditions Verdier, 2009, p. 23-27.

${ }^{2}$ André Bazin, Qu'est-ce que le cinéma ?, Paris, Éditions du Cerf/Corlet, 2008, p. 13.

${ }^{3}$ Ibid., p. 91, et aussi p. 191. 
timement s'il n'y a pas tout d'abord une certaine régression esthétique à défendre un réalisme imprescriptible du cinéma et d'ignorer la manipulation consciente par l'artiste du matériau enregistré. Bazin conçoit le film comme étant uniquement composé de documents immaculés et de fragments neutres, et pourtant il semble impossible de nier tant le fait que chaque grand film porte la signature de son auteur, que la soumission très rapide, juste après l'invention du cinéma, des formes filmiques aux genres élaborés ailleurs (l'espionnage, la farce, le burlesque). Bref, dès le départ, le cinéma a été plus qu'un certificat de présence, stencil ou index, pour être une pure fantasmagorie, une inventivité bien ancrée dans les courants de la culture et du savoir. Ce n'est donc pas un hasard si, très tôt, le cinématographe a trouvé ses beautés dans l'écriture par l'ombre et la lumière, dans sa capacité à délivrer de nouvelles formes de poésie incompatibles avec l'enregistrement brut d'une séquence spatiotemporelle. De plus, considérant la supposée reconduite par le cinématographe des conditions de perception naturelle, on objectera deux choses : la première, c'est que sous cette hypothèse, le cinéma hérite en réalité d'une vision qui n'a rien de naturel, puisqu'elle dérive en droite ligne du Quattrocento, d'une perspectiva artificialis — de ce point de vue, il y a un lien profond de la technique et de l'idéologie, qui annule l'idée du réalisme en ce que celui-ci contiendrait d'objectivé pure ; ensuite, même cette hypothèse de cinéma comme reconduite du reflet spéculaire acceptée, il est évident que, dans les faits, la pratique artistique s'est très vite débarrassée de la vision anthropocentrée et de l'œil humain, et ceci de façon multiple, comme le montrent les trois premières grandes écoles de montage, la soviétique, l'américaine et la française, qui ont toutes tenté d'ancrer la perception ailleurs que dans la vision humaine.

Bref, nous pensions être en bonne voie, et nous voilà enfermés dans les pires banalités: l'objectivité brute attestée par l'image, l'anonymat de la genèse, les faits reproduits mécaniquement. Si l'on est un tant soit peu ranciérien, on objectera qu'ainsi on déduit les propriétés ontologiques du médium à partir de ses propriétés techniques; et on fera immédiatement remarquer qu'en réalité, ces transformations de l'art dit «moderne», plus prompt à la dispersion, à la saisie des micro-événements, éloigné des grands récits, qui réunit le voulu et le non-voulu, le conscient et l'inconscient - tout ça donc est déjà préparé en amont, depuis bien longtemps, en réalité depuis le $\mathrm{XVIII}^{\mathrm{e}}$ siècle et l'invention de l'esthétique, au sens d'un certain régime d'identification de l'art. Si le cinéma a pu devenir un art et pas une simple attraction de foire, ce qu'il était au tout début, c'est précisément à cause de la révolution esthétique. Bref, il faut renverser la formule moderniste qui liait les conditions techniques à la spécificité du médium: c'est parce que le 
régime esthétique a déjà défait la corrélation entre sujet et mode de représentation, parce que «l'anonyme est devenu le sujet d'art, que son enregistrement a pu devenir art $»^{1}$. Aussi, il ne faut pas trop se laisser séduire par la conjonction qui lie le cinéma et le processus général de modification du système perceptif des hommes à l'âge des machines et du capitalisme. Outre que nous ne sommes pas convaincus par la collusion de la modernité et de la précipitation du monde (connivence qui en appelle immédiatement à l'injonction de prendre du temps pour penser nos vies devenues bonnes à rien), il semble que la conception d'un monde où le plus anodin, le plus fortuit se laisse déjà déchiffrer, la promotion des espaces quelconques et des vitesses aberrantes, ont en réalité précédé de loin les techniques d'appropriation objective du réel. La conjonction du cinéma et de la modernité est tellement imprécise qu'elle laisse tout passer entre les mailles du filet.

Mais tout cela serait à la rigueur futile si la naïveté n'était pas hantée par un grand danger, un péril cette fois-ci politique. S'il est vrai, comme l'a fait remarquer Barthes, que le rêve de tout critique, sans doute, est d'arriver à définir un art par sa technique, défendre le réalisme comme essence du cinéma semble être difficilement séparable du désir un peu répugnant d'un monde noble et primitif qui serait resté immaculé; un désir qui serait accompli au plus haut degré par cet art technique et "moderne» qu'est le cinéma, véritable litanie d'une danse des énergies s'inscrivant directement sur la pellicule, machine à imprimer la vie qui, quand elle est bien utilisée, c'est-à-dire quand son essence n'est pas dénaturée, fait du monde lui-même son vocabulaire. Toutes ces indications ne font que circonscrire le rêve le plus vieux et le plus fatigué, celui de la pureté. Or il est faux de dire que la caméra ne peut pas mentir; bien souvent, elle appartient en réalité aux dominants, si bien que l'enthousiasme pour le cinématographe comme ce qui rend naturellement le réel contient toujours l'idéologie comme son impensé. En cela, l'appareil ne parachève qu'un certain réalisme, celui déterminé par les rapports de production capitaliste, ses objectifs économiques et idéologiques. Ces remarques anecdotiques et simples permettent de ne pas perdre de vue néanmoins la question de la technique dans son alliance avec l'industrie et l'ordre de la domination, nous rappelant qu'il y a quelque équivoque idéologique à certifier pour les arts mécaniques un accès non médiatisé au Réel en tant que tel, à clamer un lien direct et antéprédicatif du film à

\footnotetext{
${ }^{1}$ Jacques Rancière, Le Partage du sensible, Paris, Éditions La Fabrique, 2000, p. 4849 ; Id., La Fable cinématographique, Paris, Éditions du Seuil, 2001, p. 16: «Le cinéma comme idée de l'art a préexisté au cinéma comme moyen technique et art particulier ».
} 
l'être même des choses. Bref, un grave problème émerge, celui du vœu pieux du cinématographe à son supposé « réalisme congénital » ${ }^{1}$. Tout ça est encore trop proche du désir de retrouver le natal, de la nostalgie béate de la primitivité et de la consistance des choses, le film trouvant dans cette intimité à l'être même l'une de ses prédispositions techniques, sa fondation ultime. Sur ces points, les réalistes ont toujours un réel de retard. Cette injonction à s'aligner sur les normes du code réaliste a été patiemment analysée par Bourdieu, qui, dans son étude sur la sociologie de la perception esthétique faisait remarquer :

Une œuvre apparaît comme « ressemblante » ou « réaliste » lorsque les règles qui en définissent les conditions de production coïncident avec la définition en vigueur de la vision objective du monde ou, plus précisément, avec «la vision du monde » du spectateur, c'est-à-dire avec un système de catégories sociales de perception et d'appréciation qui sont elles-mêmes le produit de la fréquentation prolongée de représentations produites selon ces mêmes règles ${ }^{2}$.

Ces remarques sommaires suffisent à récuser sur plusieurs plans (technique, esthétique, politique) l'affirmation du cinématographe comme art réaliste. Mais peut-être que dès le début, notre problème a été mal posé, puisqu'il a toujours consisté à saisir ce supplément, surplus ou surcroît de réalité atteint par le cinéma en comparaison à d'autres formes d'art. On pourrait évidemment se demander si Bazin lui-même n'a pas entretenu ce malentendu, par exemple à travers l'usage constant d'expressions métaphoriques et quelque peu surfaites : " épiphanie du réel », "vocation réaliste du cinéma », "réalisme congénital du cinéma », le cinéma est la «dramaturgie de la nature $»^{3}$. Mais, comme disait Deleuze, c'est le destin de la ruse, que de paraître trop naïve à des naïfs se croyant trop savants. Aussi, une lecture attentive de ce postulat ascétiquement réaliste résiste à la simplification, et l'idée même d'un

${ }^{1}$ André Bazin, Qu'est-ce que le cinéma ?, op. cit., p. 139.

2 Pierre Bourdieu, «Sociologie de la perception esthétique», dans Bernard Teyssèdre (dir.), Les Sciences humaines et l'œuvre d'art, Bruxelles, Éditions La connaissance, 1969, p. 174.

${ }^{3}$ André Bazin, Qu'est-ce que le cinéma ?, op. cit., p. 164. De façon plus circonstancielle, on peut évoquer aussi une certaine amplification de l'importance de l'article «de jeunesse» qui ouvre le recueil («Ontologie de l'image photographique »), mais qui ne nous semble devoir acquérir aucune priorité. À la rigueur, la meilleure démonstration que là n'est pas l'essentiel est l'héritage de Bazin luimême, la « dette symbolique » non remboursable que lui doivent Comolli, Daney, et quelques autres. 
retour niais aux choses mêmes chez Bazin devient trompeuse, car il n'y a chez lui aucun mysticisme, mais simplement l'affirmation que ses disciples surent redécouvrir que « la machine-caméra filme des sujets et des corps bien réels ${ }^{1}$, avec ce que cela implique de croyance aux yeux des spectateurs. Remarquons que cet énoncé est fort différent de celui du réalisme naïf : « Le cinéma est la reproduction mécanisée du réel ».

Néanmoins, nous ne pouvons pas encore saisir l'enjeu véritable de cette souscription obsessionnelle de Bazin au réalisme, son cri en ce que celui-ci contient d'exigence que les structures formelle et politique de l'œuvre soient inséparables. Les conclusions précédentes n'avaient ainsi de valeur que provisoire, car le problème n'a trait ni à la genèse automatique des apparences, ni à la capacité du cinéma à rendre parfaitement le réel, à capter dans sa durée une réalité préalable et extérieure, encore moins à reconduire le modèle de la perception de l'œil humain. Tout le monde sait bien que le représenté n'est pas le réel. Certes l'image de cinéma renvoie, dit-on, analogiquement à la chose vue ; mais il y a toujours un écart, une « marge de traduction » due au fait que - comme le soulignait avec humour Cavell les choses n'aient pas de vues ou de visions, de la même manière qu'elles ont un volume, une odeur ou une sonorité par exemple ${ }^{2}$. En ce sens, la reproduction mécanisée ne consiste pas à créer des copies, puisqu'à la rigueur, il n'y a même pas d'original dont la copie pourrait être la copie. Sans doute cela parait-il simple, mais peut-être que nous avons oublié l'étrangeté du mode de donation de la trace au cinéma, de l'image comprise comme analogon, et de la difficulté de la placer ontologiquement. Car dire que le représenté n'est pas le réel, c'est dire dans un double mouvement que, d'une façon ou d'une autre, le cinéma fait partie de la réalité, qu'il est fait du réel, mais aussi qu'il le module, qu'il le travaille. Analogie, ressemblance, similarité, semblance, sont alors autant de termes qui rentrent dans un réseau où il n'est plus possible de les identifier.

Du coup, le questionnement du réalisme cinématographique se transforme, et s'interroger sur la part de réel au cinéma revient à s'interroger sur l'autorité morale des images, sur leur capacité à être consommées politiquement, et non simplement esthétiquement. En effet, contre l'explication répandue qui ne tient nettement compte que des présupposés technicistes sur l'enregistrement et de l'épiphanie des «faits bruts » que les nouveaux

\footnotetext{
${ }^{1}$ Jean-Louis Comolli, Corps et cadre. Cinéma, éthique, politique, Lagrasse, Éditions Verdier, 2012, p. 157.

2 Stanley Cavell, The World Viewed, London/Cambridge (Mass.), Harvard University Press, 1979, p. 20.
} 
médias sont censés procurer, il faut soutenir que Bazin a conçu le réalisme moins comme la possibilité du cinéma de transmettre la consistance spatiotemporelle du monde, mais bien plutôt comme une norme pour aborder la politique de l'image. Dès lors, à la sortie de la guerre, l'interrogation qui parcourt l'œuvre critique de Bazin ne porte que marginalement sur l'ontothéologie de l'image photographique et concerne davantage l'attitude du cinéaste face à un médium ayant des affinités spécifiques vis-à-vis du réel. Car si l'œil mécanique est capable de tout digérer, tout capter, cette incorporation du monde par la caméra atteste aussi une forme de violence proprement cinématographique.

Nous demandons donc maintenant : quel genre de rapport les images dites « réalistes » entretiennent-elles réellement au réel ? Que peuvent-elles dire sur la responsabilité de l'artiste et l'engagement du cinéaste auprès du monde? En quel sens les formes de l'art sont-elles aussi une forme de rapport au monde ? C'est grâce à ces interrogations que plusieurs générations de cinéastes et de théoriciens ont compris par "réalisme», à la suite de Bazin, le fait que les problèmes moraux et esthétique sont d'un seul tenant, et que la forme d'une cuvre d'art est la manifestation de sa structure éthique une position bien résumée dans la formule devenue célèbre de Godard : «Le travelling est une affaire de morale ». Ce pourquoi aussi, sous couvert de parler de cinéma, Bazin a surtout et avant tout parlé de la façon que nous avons de nous orienter dans le monde. Écrire sur le cinéma n'était pour lui rien d'autre qu'écrire sur le monde: en parlant du cinéma, on parle en définitive de tout, on arrive à tout. C'est pour cela aussi que les Cahiers $d u$ cinéma défendaient l'idée aujourd'hui saugrenue que ça valait la peine de penser le cinéma.

Mais il n'est toujours pas établi toutefois pourquoi la posture éthique du cinéaste doit nécessairement passer par la promotion du réalisme et par le refus de l'esthétisation. Le cinéma, a dit Bazin, "sauve l'être des apparences ». Mais l'ambivalence sémantique du verbe « sauver» le place à la fois du côté de ce qui délivre, révèle ou prend soin de, et du côté de l'arrêt par le biais d'une fixation, d'une captation ou d'une capture - quand nous disons "prendre » une photo, prendre est aussi bien voler, extorquer, saisir. Couvrir un événement, c'est à la fois l'aborder, s'impliquer, mais aussi le dissimuler, l'éviter. Si l'on prend la pleine mesure de cette violence intrinsèque du médium, on entrevoit que le réalisme ne s'oppose pas à ce qui, dans le film, serait irréel : l'onirique, le ludique, le fantastique. L'opposé du réalisme est le formalisme, en ce que celui-ci contient d'inconséquent à la présence authentique au monde. On retrouve ici l'antagonisme des deux 
tendances bien connues depuis Kracauer ${ }^{1}$, un antagonisme qui prend luimême racine dans la conscience que l'enregistrement peut vite tourner en une affaire abjecte. L'acte de filmer est trop facile, trop rapide, trop automatique précisément, c'est-à-dire beaucoup trop proche de l'effervescence inconsciente, de l'idéologie la plus puante, pour ne pas en appeler à une certaine méditation des images qui aurait pour fonction de moraliser des actes fondamentaux du cinéaste. La caméra capte les émanations lumineuses des choses et des êtres, mais à travers ce transfert de réalité, à la fois elle protège les apparences en en créant une empreinte, et en arrêtant le cours du temps, en le gelant, elle est la mort elle-même au travail, une relique ou un masque funéraire. Filmer pour André Bazin revient à embaumer le temps, à momifier le devenir des choses. Le cinéma, c'est le royaume des ombres, la mort 24 images par seconde.

Cela sonne évidemment comme une ironie : il était demandé au cinéma de racheter la réalité matérielle, mais voilà que la caméra participe au processus de réification lui-même, en produisant des apparences anesthésiées, fichées, «comme des papillons $»^{2}$. D'où l'analogie souvent remarquée de la caméra et d'une arme, de l'acte de filmer et de la chasse, du caméraman et du mitrailleur, des formes sur l'écran et des spectres. Les instantanés fixent à jamais un instant précis et transitoire, ils sont une coupe arbitraire de la vie, et en cela comparables à une forme de meurtre : apparences captées, meurtries, qui revivent pourtant sur l'écran. Eu égard au cinéma, cette contradiction n'est pas simplement circonstancielle, elle devient le point nodal des discours sur la politique des images, où le film est tantôt un agent de révélation (Errettung, sauvegarde, préservation), tantôt d'agression, et ce qu'on ressent alors devant les traces matérielles, c'est plutôt la capacité de la caméra de violer, d'engloutir, voire de digérer tout sur son passage, en prenant des instantanés comme si elle voulait avoir un bref aperçu des êtres et des choses avant que ceux-ci ne s'évanouissent.

Ce double aspect du cinématographe n'est pas indifférent à l'apologie du réel qu'on trouve chez Bazin. La nature même de l'enregistrement, prédatrice et violente, exige de chaque cinéaste d'être conscient que son travail découpe et émiette le monde. Deux possibilités se présentent face à ce morcellement : soit cette violence n'est pas affrontée, et alors le film devient

${ }^{1}$ Siegfried Kracauer, Théorie du film. La Rédemption de la réalité matérielle, trad. fr. D. Blanchard et C. Orsoni, Paris, Éditions Flammarion, 2010. Voir aussi dans le présent volume le texte de Maud Hagelstein et Jérémy Hamers.

${ }^{2}$ Roland Barthes, La Chambre claire. Note sur la photographie, Paris, Éditions du Seuil, 1980, p. 90. 
affaire de diégèse, de drame bien construit où les significations sont plaquées sur les apparences, il est affaire de représentation imaginaire des créatures auxquelles il n'arrive que des aventures de fantaisie — c'est ce formalisme-là qui a tant dégouté la «bande à Bazin »; soit il prend garde à cette violence et, au lieu de prétendre de réorganiser les fragments en une totalité inatteignable, l'artiste résiste au caractère potentiellement infâme et honteux d'une telle reconstruction. À partir de ce point de «non-tricherie», une masse de ficelles dramatiques et de procédés formalistes sont impossibles : le schéma sensori-moteur peut vaciller, les grandes histoires font désormais intervenir la poussière des micro-événements, l'intérêt n'est plus ramené sur l'héroïne, les images cessent d'être lisses et prennent tout en écharpe, l'écran de cinéma cesse d'être centripète pour devenir centrifuge, cache plutôt que cadre. Mais là encore, il y aurait quelque frivolité à dire que pour Bazin la chose était claire, que c'est le néoréalisme italien qui doit nécessairement servir de mesure : soit l'artiste utilise l'empirique à ses fins personnelles (même « esthétiques ») à travers une série de procédés formels abjects, soit il explore la réalité pour elle-même. La disjonction exclusive a toujours la monotonie de ce qui rapproche tout en voulant séparer. Comme si, dans un cas, le cinéaste créait nécessairement une vérité rhétorique, artistique, qui n'appartient pas aux apparences elles-mêmes, tandis que dans l'autre cas il y aurait une « saisie intuitive de ce qu'il faut, comme il faut ${ }^{1}$, un tact naturel où les événements filmés seraient respectés et recèleraient un sens qui leur est propre, si bien que le monde serait dit " sans dicter ce qu'on a à en dire », comme si le filmé imposait de lui-même ses lois de représentation.

Là encore, on gagnerait à reformuler le problème en des termes plus stricts : l'antiformalisme de Bazin vise moins une croyance béate dans l'aptitude du cinéma à rendre le réel, mais découvre le réalisme comme la vérité d'un rapport, du lien du cinéaste à un réel qu'on suppose pouvoir échapper à la violence d'une signification simplement appliquée sur les événements représentés. Un enjeu se dégage là, disait Serge Daney, car alors l'enregistrement possède immédiatement une dimension morale. La beauté n'est que le gage ou la prime récoltée pour cet héroïsme moral. Le cinéaste photographie des rapports et non des choses ; car il faut du réel pour servir de «toile de fond » et d' « écran d'arrêt » à l'émergence d'un objet moral : précisément l'attitude, la posture ${ }^{2}$. C'est ce rapport qui doit faire l'objet du plus grand travail, et la vérité ne peut être que celle de ce rapport (ainsi Peter Watkins

\footnotetext{
${ }^{1}$ André Bazin, Qu'est-ce que le cinéma ?, op. cit., p. 276.

${ }^{2}$ Serge Daney, L'Exercice a été profitable, Monsieur, Paris, Éditions P.O.L, 1993, p. 81 .
} 
dans son travail sur La Commune (Paris, 1871)). Ce qui compte donc, c'est moins ce qu'on filme que le dispositif d'enregistrement, les résultats auxquels il parvient et les procédés qu'il offre. Et l'enseignement de Bazin qu'on a le plus retenu n'est-il pas finalement que l'image doit être la trace matérielle d'une rencontre, d'un corps à corps (et donc (1) que la critique d'un film est inséparable de la critique de ses conditions de production, et (2) que tout film est un documentaire sur son propre tournage) ?

Le problème connait ainsi une singulière altération: une machine et des corps partagent une durée qui est faite de leur conjonction. Il faut alors s'exposer, se risquer, de se frotter à l'autre ${ }^{1}$. Contre une œuvre balisée, un imaginaire appauvri, acclimaté, contre les significations conventionnelles, assainies, nettoyées de leurs dangers, contre les objets déjà tout désignés, contre les énoncés déjà formés et formulés, Bazin affirme que s'acharner contre le réel, faire en sorte qu'à l'image il résiste et insiste, c'est l'utopie du cinéma. Ce n'est pas la soumission de l'artiste à ce qui vient à lui, mais une contrainte qui rend possible la lutte par laquelle passe tout acte de création ; l'expérience du négatif. Avec la ténacité du réel, on ne peut pas filmer tout ce qu'on veut, comme on veut, on ne peut pas jouer avec les images. Enfin, nous découvrons quelque chose qui ne dépend plus de notre volonté. Bref, refuser le maniérisme, c'est attester qu'il y a toujours un reste qui échappe au transformateur esthétique introduit par la reconstruction et à l'organisation souveraine imposée par le scénario. "Il y a une part maudite, laissée en rade $»^{2}$, qui échappe à la simple syntaxe de l'intérêt et à la dramaturgie des grands sujets. Plutôt que démontrer, il suffit pour les réalistes déjà de montrer, à condition de préciser que montrer est en fait le plus difficile. Montrer, c'est comprendre que l'image compte d'abord « non pour ce qu'elle ajoute à la réalité, mais pour ce qu'elle en révèle ${ }^{3}$. Et finalement n'est-ce pas là la destination de tout art, celle de dégager la valeur et la vérité de la $\operatorname{vie}^{4}$ ?

«Dans le découpage cinématographique habituel [...] le fait est attaqué par la caméra, morcelé, analysé, reconstitué : il ne perd sans doute pas sa nature de fait, mais celle-ci est enrobée d'abstraction ${ }^{5}$. Suspendre cette imposition abstraite du sens devient alors l'opération et la tâche la plus

${ }^{1}$ Jean-Louis Comolli, Corps et cadre, op. cit., p. 242. et p. 175.

${ }^{2}$ Sur ces questions, $c f$. les pages fédératrices de Serge Daney, L'Exercice a été profitable, Monsieur, en particulier p. 117.

${ }^{3}$ André Bazin, Qu'est-ce que le cinéma?,op. cit., p. 67.

${ }^{4}$ Gilles Deleuze, Proust et les signes, Paris, PUF, 2010, p. 45.

${ }^{5}$ André Bazin, Qu'est-ce que le cinéma?, op. cit., p. 280. 
difficile, la plus risquée, exigeant à la fois une très grande technique et une loyauté intellectuelle totale ${ }^{1}$. Pour Bazin, il y a danger à filmer, et c'est là son réalisme : la promotion d'un art où rien n'est innocent, où l'image importe, compte, nous regarde aussi bien. Ce n'est pas simplement une façon de faire qui serait différente, c'est une question d'honnêteté et d'intégrité. Les tricheurs, les menteurs, les petits esprits qui ne compromettent personne, qui ne supportent que les déterminations explicites, qui ne peuvent que décomposer et triturer ce qui ne demande pourtant qu'à faire l'objet d'une rencontre : voilà les ennemis jurés du cinéma, ceux par qui il se dégrade et replonge dans le règne de la bêtise. Pour Bazin, il s'agit de tordre le cou au formalisme et d'atteindre à l'incalculable, au non programmable, au non encore maitrisable, pour que le monde représenté ne soit que partiellement domestiqué, et pas tout entier versé dans l'idéologie, l'industrie, un spectacle déjà mis en scène, la petite mécanique des clichés et des habitudes prises ${ }^{2}$.

Et pourtant, cet antiformalisme ne nous donne encore aucune garantie, tout ça paraît être encore trop facile, trop simple, uniquement basé sur la structure apparente du réalisme, sans que celui-ci ait pu recevoir sa nécessité d'ailleurs. Nous avons prétendu que le réalisme consistait au cinéma en de simples procédés et trucages formels qui minimisent l'emprise de l'artiste sur son matériau. Et pourtant, il doit être conquis et gagné dans une lutte contre la stupidité, qui est aussi la bêtise de la pensée. La " justesse » du réalisme, c'est que dans la descente vers l'obscurité, engloutis dans l'écran, anonymes et contemplant, solitaires, un monde en l'absence d'homme, nous redécouvrions la lumière comme forme de la pensée, un monde qui, au hasard des rencontres, cherche à être déchiffré.

Même si cette conclusion pour Bazin suppose un voyage long et sinueux dans ses textes et dans ceux de ses disciples-héritiers, il est devenu clair que le problème concerne avant tout la politique de l'image. Parce que filmer n'est pas innocent, dès que l'homme prend la décision de filmer telle chose et non telle autre, à un moment et pas à un autre, et de combiner ses images suivant un certain ordre, il s'oriente dans la pensée, d'une tout autre façon que le réalisme naïf défendu plus ou moins désespérément par ceux qui déduisent les propriétés ontologiques d'un art de ses propriétés techniques. On comprend alors le violent rejet d'une imposition superficielle de la signi-

${ }^{1}$ Roland Barthes, «Entretien avec Roland Barthes », Cahiers du cinéma, 2001, p. 53.

${ }^{2}$ Jean-Louis Comolli, «Filmer le réel ? », dans Id., Corps et cadre, op. cit., p. 292293, et l'influence manifeste de Bazin, notamment sur «la charge d'ambiguïté supplémentaire $»$. 
fication. L'instant décisif n'est pas simplement le fruit du hasard, mais doit devenir la rencontre qui nous force à penser. Bazin prend au sérieux l'idée qu'un certain mode de pensée au cinéma détermine les modes dont apparaît l'enregistré. Tout manque au niveau de la forme réfère nécessairement à un manque au niveau de la pensée. Mais dans quoi se meut la pensée ? Car si l'élément de la nage est l'eau, comme dirait Heidegger, nous ne savons pas encore quel est l'élément de la pensée, et ici la pensée au cinéma. Ce n'est donc pas aux autres artistes que chaque cinéaste doit être comparé, mais c'est plutôt chaque grand artiste qui doit être comparé à un penseur ${ }^{1}$ : tous portent en eux comme un ton ou style, nécessairement signés.

Cette opposition à la bêtise de la pensée au cinéma connaît chez Bazin et ses héritiers trois aspects majeurs, qui ne sont séparables que dans l'abstraction: (1) la volonté de déstabiliser les images du pouvoir, les récits normés et les représentations idéologiques; (2) l'affirmation du manque intrinsèque de toute vision, toujours aveugle à ses propres bévues, si bien qu'il faille toujours que l'image pointe ses propres conditions de production, il faut que l'énonciation se fasse sentir sous l'énoncé ; enfin, (3) filmer ne peut pas se faire sans l'expérience du négatif. Cette triple opposition au formalisme renvoie évidemment à la lutte de l'image contre le cliché chez Deleuze $^{2}$; ou encore au dégoût de Barthes face à l'image unaire qui copie le réel sans le doubler, sans le faire vaciller, sans rendre sensible la présence d'une tache aveugle à même le visible ${ }^{3}$; elle renvoie, enfin, au refus de Daney d'avaliser le naturalisme, qu'il définit comme suit: "Technique qui reconduit quelque chose qui lui préexiste : la société en tant qu'elle est déjà une mise en scène ». Par opposition, « travailler ce donné, casser cette prémise en scène, la rendre visible en tant que telle, est toujours une entreprise courageuse, difficile, impopulaire. Le réalisme est toujours à gagner $»^{4}$. Il s'agit vraiment d'un ascétisme, d'une « écologie des images » qui ne contre-

\footnotetext{
${ }^{1}$ Sur ce thème, la référence est évidemment Gilles Deleuze, Différence et répétition, Paris, PUF, 1968, p. 169-217 ; Id., Proust et les signes, op. cit., p. 115-124 ; Id., Nietzsche et la philosophie, Paris, PUF, 1962, p.118-126; mais aussi Martin Heidegger, Qu'appelle-t-on penser ?, trad. fr. A. Becker et G. Granel, Paris, PUF, 1954 ; Id., "Que veut dire "penser" ? », dans Id., Essais et conférences, trad. fr. A. Préau, Paris, Éditions Gallimard, 1958, p. 151-169.

${ }^{2}$ Gilles Deleuze, L'Image-mouvement, Paris, Éditions de Minuit, p. 289: «De l'ensemble des clichés doit sortir une image. Avec quelle politique et quelles conséquences ? »; Id., Francis Bacon. Logique de la sensation, Paris, Éditions du Seuil, 2002, p. 83-85.

${ }^{3}$ Roland Barthes, La Chambre claire, op. cit., p. 69-71 et p. 89-84.

${ }^{4}$ Serge Daney, La Rampe, Paris, Éditions Gallimard/Cahiers du cinéma, 1996, p. 61.
} 
dit pourtant pas l'exubérance et la fantaisie (il n'y va jamais chez Bazin d'une nouvelle Inquisition, où il s'agirait de juger tout film à l'aune de préceptes ou d'un manifeste esthétique : c'est au contraire chaque film qui doit dicter la loi de sa propre évaluation, comporter sa propre critique). Éthique, cinéma, politique ne sont même plus des domaines séparés dont il s'agirait de se demander s'ils doivent être mis en contact. Une manière basse de penser, la paresse, l'évitement des rencontres ne produiront jamais aucune grande œuvre, mais ne feront que reconduire l'ordre de la domination, sans même chercher à le faire. D'où la méditation patiente de Comolli sur l'état actuel du cinéma, quid facto et non plus quid juris : pourquoi le spectacle irréalise-t-il à ce point le monde ? Pourquoi, aujourd'hui encore, le cinéma, un art pourtant vanté pour sa reproduction fidèle de la réalité, est de moins en moins au centre de nos réalités, alors même que l'industrie hollywoodienne les a infiltrées et modelées depuis plus d'un siècle?

Ces brèves remarques sur l'art de résister aux images permettent de produire une analyse polarisée. D'une part, parce qu'elle émerge du déni de tout supplément, de toute hantise, de tout manque ou de toute latence, l'image figée qu'est le cliché est frontale, elle est tout le contraire d'une rencontre : elle n'admet de beauté que classée, c'est la réalité domestiquée, codée et enrobée d'abstraction. Cette réalité rabotée et euphémisée a alors entièrement basculé du côté de la représentation. Ne se poser que des problèmes formels esthétiques ou ne s'en poser aucun revient au même : toujours la peur généralisée d'un signe ou d'une rencontre comme genèse de l'acte de penser. En ce sens, naturalisme et publicité ont plus en commun qu'ils n'ont de différences, ils sont déjà compromis. Contrairement au cliché qui cloisonne, nivelle, banalise, qui commande, apprivoise, qui fixe l'attention et organise, qui emprisonne, cadre et encadre une réalité qui n'est même plus récalcitrante, l'image - elle - refuse de maîtriser le réel, elle résiste à la facilité d'en faire une proie, elle nous dé-familiarise, elle démarque $^{1}$. En ce sens, elle est tout l'inverse d'une image de marque. Et c'est précisément parce qu'ils sont si attendus, si dociles, tellement signifiants, parce qu'ils ne tiennent à rien, que les clichés se prêtent à tous les jeux de montage et ne servent que les puissances établies, qu'ils sont incapables de résister aux manipulations. Dans tous les cas, le précepte des disciples de Bazin est toujours le suivant : quelque chose doit devenir « trop fort dans l'image $»^{2}$, quelque chose doit déborder, être moins disponible, plus sauvage,

${ }^{1}$ Ibid., p. 57.

${ }^{2}$ Gilles Deleuze, L'Image-temps, Paris, Éditions de Minuit, 1985, p. 29. 
bref, plus insignifiant, c'est-à-dire davantage du côté du réel ${ }^{1}$. Là où le formalisme rend les choses en tant qu'elles sont déjà modulées et préformées (natura naturata), l'image authentique délivre les forces par lesquelles les choses et les êtres prennent forme sous nos yeux (natura naturans) : "L'impression d'un ordre naissant, d'un objet en train d'apparaître, en train de s'agglomérer sous nos yeux $»^{2}$. Le salut du cinéma passe par la découverte de cette part non normée sous le cliché, ainsi la fameuse scène décrite par Deleuze qui marque la rupture sensori-motrice : la rencontre d'un regard, d'un ventre de jeune bonne enceinte, et de toute la misère du monde (Umberto $D$, de De Sica) ${ }^{3}$ : remontée des situations optiques et sonores pures qui font de nous des voyants.

Placer, comme Bazin, Comolli, Daney ou Deleuze le font, l'image du côté de la vérité de l'acte de montrer, de la rencontre, et renvoyer le formalisme d'emblée du côté de l'image de marque, de marketing et du pouvoir, implique qu'il y ait nécessité pour la caméra de distinguer qu'elle ne peut tout couvrir à la fois, pour qu'elle puisse s'assurer de réellement discerner ce qu'elle choisit de regarder, et de spécifier les conditions d'un tel regard. Les cinéastes ne doivent pas seulement voir et entendre (ce qui déjà en soi n'est pas toujours facile), mais aussi voir et entendre les limites mêmes de leur audition et de leur vision, pour que l'image préserve un résidu indéfini qui excède toute totalisation. Bref, comme disait Didi-Huberman, commentant la poétique de Brecht, «montrer que l'on montre, c'est ne pas mentir sur le statut épistémique de la représentation ${ }^{4}$. L'image, ou plutôt quelque chose dans l'image, embarrasse : il y a un reste, une irréductibilité, l'impossibilité de servir une cause ou une signification préformée. Quand Bazin félicite les grands cinéastes de coudre «la robe sans couture de la réalité », il ne s'agit pas pour lui «de clamer qu'il faut laisser le monde intact, mais au contraire qu'il faut lui poser question, le troubler $»^{5}$. Une fois pris dans ce combat contre la prolifération d'images (qu'on ne dira même pas irraisonnée, mais au contraire trop raisonnable), le film ne peut pas exercer d'effets d'imposition, il ne peut pas être unilatéral, univoque ou monologique, il doit désapprendre, retrancher, pour retrouver l'image sous le cliché.

\footnotetext{
${ }^{1}$ Sur cet « effet de chair », aussi appelé « effet de réalité », $c f$. Jean-Louis Comolli, Corps et cadre, op. cit., p. 110-111.

2 Maurice Merleau-Ponty, «Le Doute de Cézanne», dans Id., Sens et Non-sens, Paris, Éditions Gallimard, 1996, p. 25.

${ }^{3}$ Gilles Deleuze, L'Image-temps, op. cit., p. 8.

${ }^{4}$ Georges Didi-Huberman, Quand les images prennent position, L'Eil de l'histoire I, Paris, Éditions de Minuit, 2009, p. 67.

${ }^{5}$ Jean-Louis Comolli, Corps et cadre, op. cit., p. 73.
} 
Il s'agit vraiment d'une nouvelle conception du visuel, mais une conception exprimée sur le mode d'une guerre, d'une lutte ou d'un combat contre la bêtise du formalisme ${ }^{1}$. Le cinéma réaliste trame ainsi un univers où le réel freine encore un peu plus sa propre manipulation, où il échappe à sa propre mise en forme. L'essentiel est que l'artiste fasse l'expérience du négatif, la seule manière à la caméra de fournir des images authentiques, de «cogner contre ce qu'elle filme $»^{2}$.

Quand, dans son texte fédérateur, Rivette décrivait le travelling abject de Kapo, il était héritier de cette esthétique bazinienne qui posait que le cinéaste juge ce qu'il filme et devrait être jugé suivant la façon dont il filme ${ }^{3}$. Ce qui se dessine en creux dans cette obsession du réalisme, ce combat contre l'abjection, c'est la perspective où il devient impossible de "distinguer la forme esthétique de son contenu éthique ${ }^{4}$, où l'on ne peut plus prétendre que le cinéma ne connait de rapports que techniques, et donc qu'il rend simplement le réel, au lieu de le rendre problématique. Prendre la pleine mesure de ce problème, c'est se confronter à ce qui résiste au spectacle généralisé. Le monde face à la caméra décrit par Bazin est toujours supposé exercer une force d'opposition, il n'est pas sous la main, parfaitement disponible, simple stock ou réserve; il résiste à sa propre réduction en refusant toute forme de sur-sophistication ${ }^{5}$, de jeu vide, c'est-à-dire de jeu simplement sur l'image, et non sur les événements, les choses, les peuples, les classes. Au cinéma - du moins dans l'utopie politique qu'il porte chez les plus grands cinéastes - quelque chose de rebelle résiste, c'est la butée du réel, un reste qui à la fois nous échappe et qui nous saisit.

Créer une image sans fragmentation, totalisante, désignant directement un contenu, remarquait Didi-Huberman à propos des instantanés recardés, retouchés d'Auschwitz, c'est insulter le danger, c'est faire de la condition d'urgence de ce corps à corps un simple document informatif. Cette manipulation-là n'est pas simplement formelle, mais éthique et ontologique ${ }^{6}$.

${ }^{1}$ Gilles Deleuze, L'Image-mouvement, op. cit., p. 284-290 ; Id., L'Image-temps, op. cit., p. 33.

${ }^{2}$ Serge Daney, La Maison cinéma et le monde, vol. I, Paris, Éditions P.O.L, 2001, p. 201.

${ }^{3}$ Jacques Rivette, «De l'Abjection », dans Antoine de Baecque (dir.), Théories $d u$ cinéma, op. cit., p. 37-40.

${ }^{4}$ Georges Didi-Huberman, Quand les images prennent position, L'Eil de l'histoire I, op. cit., p. 107.

5 André Bazin, Qu'est-ce que le cinéma?, op. cit., p. 325.

${ }^{6}$ Georges Didi-Huberman, Images malgré tout, Paris, Éditions de Minuit, 2003, p. 50-54. 
Parce que quand l'expérience de négativité ou de résistance (Widerstand) est absente, nous sommes présents à des corps volatiles, subtilisés, privés de réalité, nous sommes face à des créatures de fantaisie, déjà normés comme des corps souffrants, belles victimes toutes prêtes pour les murs des musées ou les manuels d'histoire. Comment ne pas trembler devant un être humain qui meurt? Tout n'est pas possible, tout n'est pas filmable n'importe comment ${ }^{1}$. La question est toujours la même : comment parvenir à une image nécessaire ? Non pas du tout à la nécessité de filmer mais, dans ce qu'on filme, à un résultat qui préserve les "griffes de la nécessité ». Le désir d'une œuvre sur laquelle ne pèserait pas "l'hypothèque de la liberté d'interprétation de l'artiste $»^{2}$ n'a pas d'autre sens : «Pas de la belle photo, pas de belles images, mais des images, de la photo nécessaires $»^{3}$.

La prétendue démission du montage et du morcèlement par Bazin ne se comprend qu'à l'aune de cette interdiction. Plutôt que de refus du montage, on dira qu'il s'agit de sa réévaluation, le cinéaste devant être celui qui expose les événements en les désorganisant, donc en les sortant de leurs évidences. Il faut toujours retoucher le réel avec du réel ${ }^{4}$. Ce qu'il s'agit d'éviter est la " simple représentation imaginaire ${ }^{5}$, un découpage qui n'est commandé par aucun aspect de la réalité. Tout se passe comme si, sur le point même d'accomplir son exactitude, l'index devait concéder son impuissance dernière, son incapacité à tout saisir : "La réalité [...] échappe toujours de quelque côté $»^{6}$. Ainsi, la tâche du cinéaste est d'être le gardien d'un monde qu'on doit supposer non totalisable et irréductible à sa captation, à la manière de l'Unumgängliche heideggerien, cet incontournable que les sciences les plus hystériques n'arriveront jamais à cerner entièrement. Ici, c'est la vision qu'il s'agit d'amener à ce manque, et de l'ancrer dans ce manque $^{7}$, afin que de l'ensemble des clichés puisse sortir une image. Cette affirmation de la précarité de la vue n'est pas une posture, un effet de style ou une note d'intention qu'on pourrait ranger dans un manifeste esthétique. C'est le postulat que la caméra ne peut pas épuiser l'événement et en faire le tour, que le regard est toujours une question de relation, et en ce sens

\footnotetext{
${ }^{1}$ Serge Daney, L'Exercice a été profitable, Monsieur, op. cit., p. 107.

${ }^{2}$ André Bazin, Qu'est-ce que le cinéma ?, op. cit., p. 23.

${ }^{3}$ Robert Bresson, Notes sur le cinématographe, Paris, Éditions Gallimard, 1988, p. 92.

${ }^{4}$ Cf. André Bazin, Qu'est-ce que le cinéma ?, op. cit., p. 55 sur la destruction et la renaissance de la réalité cinématographique dans son lien à la réalité documentaire.

${ }^{5}$ Ibid., p. 59.

${ }^{6}$ Ibid., p. 272.

${ }^{7}$ Ibid., p. 312-313.
} 
lacunaire. Ne jamais montrer tous les côtés des choses, préconisait Bresson ${ }^{1}$. En cela, la tendance qui rend au film le sens de l'ambiguïté du réel laisse le spectateur lui-même sélectionner ses zones d'intérêt (ce qui ne veut pas dire qu'il pense ce qu'il veut), et donc le laisse « actif et inquiet, en alerte, sur le bord d'être perdu $»^{2}-$ n'est-ce pas là une description parfaite du spectateur émancipé ? L'opération consiste à insister sur l'arbitraire du fragment dans l'acte même de l'enregistrement. Ce qui compte, c'est l'étincelle dans l'image qui lui fait perdre tout contrôle, comme si la réalité filmée répliquait contre sa codification et sa mise en forme. Ce n'est pas la promotion du dérèglement, mais la conscience de l'arbitraire de la coupe dans la brève indication de la part qui est exclue. Faire sentir l'énonciation sous l'énoncé, en renonçant par avance au "reflet objectif», est alors toujours pour le réalisme une façon de ne pas être à la bonne place, car c'est toujours dans le décalage et le dédoublement que se niche la possibilité d'éviter l'image unaire et d'aiguiser le regard.

À travers l'affirmation de l'expérience du négatif, de la nécessité de ne pas mentir sur le statut épistémique de la représentation et du besoin de lutter contre les clichés, nous voyons qu'il n'y a plus rien de niais dans un tel réalisme. C'est même précisément cette "puissance de vue » qui fait la beauté des films de Harun Farocki, Chris Marker ou Johan van der Keuken. Chez Bazin, cette puissance découle essentiellement du refus d'avaliser un art fondé sur l'oppression ou le mépris. D'où la tonalité humaniste de son œuvre, qui semble être tout entière ancrée dans cet amour ou cette estime du réel, qu'on ose enfin affronter au lieu de le fuir, de le coder ou de le faire tenir tranquille. Et c'est précisément ce que faisaient les néoréalistes, quand ils évoquaient l'étincelle de respect ou d'amour nécessaire à l'avènement de l'image. Si bien que le problème subit un étrange revirement et peut être redéfini à nouveaux frais. Au lieu de le déterminer par son réalisme congénital, Bazin affirme, commentant l'art de De Sica, que « le cinéma est l'art propre de l'amour $»^{3}:$ "L'affection que porte De Sica à ses créatures ne leur fait courir aucun risque, elle n'a rien de menaçant ou d'abusif, c'est une gentillesse courtoise et discrète, une générosité libérale, et qui n'exige rien en

${ }^{1}$ « Tout montrer voue le cinéma au cliché, l'oblige à montrer les choses comme tout le monde a l'habitude de les voir. Faute de quoi, elles paraîtraient fausses ou chiquées » (Robert Bresson, Notes sur le cinématographe, op. cit., p. 94).

2 Jean-Louis Comolli, Corps et cadre, op. cit., p. 236 et André Bazin, Qu'est-ce que le cinéma?,op. cit., p. 77.

${ }^{3}$ André Bazin, Qu'est-ce que le cinéma?,op. cit., p. 321. 
échange $»^{1}$. Autrement dit, c'est précisément la modestie de l'acte de filmer qui garantit l'authenticité et l'universalité des sentiments provoqués. Le cinéma a la possibilité d'atteindre à cet amour innocent, cette courtoisie ou tendresse dépourvue de pitié. Mais tout cela ne serait rien si, en face des images ainsi produites, nous pouvions penser ce que nous voulions :

Ce n'est pas l'acteur qui nous émeut, ni l'événement, mais le sens que nous sommes contraints d'en dégager. Dans cette mise en scène, le sens moral ou dramatique n'est jamais apparent à la surface de la réalité ; pourtant nous ne pouvons éviter savoir quel il est [...]. N'est-ce pas là une solide définition du réalisme en art : contraindre l'esprit à prendre parti sans tricher avec les êtres et les choses ? ${ }^{2}$

Souvent nous croyons qu'il faut plus d'intelligence que d'amour : toujours plus de clichés, de beautés banalisées, de vérités abstraites qui sont le fruit du « bon vouloir» du filmeur. Combien de réalisateurs tournent pour illustrer une thèse, faire passer un message ou pour éduquer, faisant ainsi du film un prestataire de service, et de l'art une chose de la communication ou de l'information. Le cinéma en revanche porte une sensualité, une affinité mystérieuse, une qualité de présence qui est capable de briser la stupidité et de provoquer l'exercice disjoint de toutes nos facultés, c'est-à-dire de faire naître violemment l'acte de penser dans la pensée. C'est donc toujours après que l'intelligence, la pensée viennent ${ }^{3}$. Veiller à cette rencontre, "ne pas trahir l'essence des choses, les laisser d'abord exister pour elles-mêmes librement, les aimer dans leur singularité particulière ${ }^{4}$, revient alors au cinéma à l'acte politique par excellence, celui qui consiste à se laisser transpercer par les événements, au lieu de vouloir les transpercer ${ }^{5}$. Rien n'est

\footnotetext{
${ }^{1}$ Ibid p. 320.

${ }^{2}$ André Bazin, Qu'est-ce que le cinéma ?, op. cit., p. 206.

${ }^{3}$ Gilles Deleuze, Proust et les signes, op. cit., p. 32 et p. 66 ; « Tournage. S'en tenir uniquement à des impressions, à des sensations. Pas d'intervention de l'intelligence étrangère à ces impressions et sensations » (Robert Bresson, Notes sur le cinématographe, op. cit., p. 43-44); "Creuse ta sensation. Regarde ce qu'il y a dedans. Ne l'analyse pas avec des mots. Traduis-la en images sœurs, en sons équivalents. Plus elle est nette, plus ton style s'affirme. (Style : tout ce qui n'est pas la technique)» (ibid., p. 61); «Le réel arrivé à l'esprit n'est déjà plus du réel. Notre œil trop pensant, trop intelligent » (ibid, p. 79).

${ }_{5}^{4}$ André Bazin, Qu'est-ce que le cinéma ?, op. cit., p. 319.

5 «Je crois que le peintre doit être transpercé par l'univers et non vouloir le transpercer » (Maurice Merleau-Ponty, L'Eil et l'Esprit, Paris, Gallimard, 1964, p. 31, citant Charbonnier).
} 
plus loin de Bazin que la défense d'un spectacle qui semble réel; c'est instaurer la réalité en spectacle qu'il souhaite: «Un homme marche dans la rue et le spectateur s'étonne de la beauté d'un homme qui marche $»^{1}$. Simplement, ce n'est pas le réel qui est ici dramatique, mais le drame (et même l'infiniment bouleversant) qui naît des hasards, de l'inattendu, des états d'attente et de réserve. Quand l'événement libère sa signification, tout un univers devient à déchiffrer, obligeant notre pensée à se mettre en mouvement dans la forêt des signes.

Finalement, faire l'apologie du réalisme, c'est glorifier cette sorte de candeur, de réserve ou de pudeur nécessaires à l'égard de ce qui est filmé, et où on perçoit un lointain écho de ce que Kant appelait la «faveur $»^{2}$ (Gunst), le refus de réduire la chose à l'objet de désir, de plaisir intéressé, à une marque ou à un code. La liberté de la chose jugée est alors l'exacte réponse à la liberté du sujet jugeant : l'apparence des formes lumineuses sur l'écran est contemplée pour elle-même, de sorte que les êtres s'y donnent à voir librement, suivant leur propre poids, à leur façon particulière. Bazin conçoit alors la distance même du cinéma et du monde comme la condition de l'apparition du monde, parce que « cette extériorité traduit un aspect éthique et métaphysique essentiel de nos relations avec le monde ${ }^{3}$, et que seule cette extériorité, ce recul ou cette mise à distance polie permet à l'apparence d'être libre, de se donner à nous dans tout son éclat. Mais cette faveur, il faut le remarquer, n'est en rien la destitution de la force saisissante des images, car elle conduit au contraire à «une morale à laquelle l'esprit ne peut précisément pas échapper parce qu'elle lui vient de la réalité même ${ }^{4}$.

Ainsi, combien triste est l'idée que l'essence réaliste du cinématographe doit être dérivée de la référentialité et de l'automatisme du médium. $\mathrm{Ne}$ sommes-nous pas finalement forcés d'admettre plutôt que ce réalisme n'est rien d'autre que la volonté de saisir le monde dans ses émanations lumineuses et sonores, émanations qui permettent à l'art de « démasquer une nature qui lui ressemble $»^{5}$ en faisant paraître ce qui ne serait peut-être jamais vu? Ce n'est pas un spectacle qui serait fait du réel, mais la réalité elle-même instituée en spectacle. Sur l'écran tout fleurit de nouveau, comme ces fleurs de papier japonaises plongées dans l'eau vive, afin que la vie soit

\footnotetext{
${ }^{1}$ André Bazin, Qu'est-ce que le cinéma ?, op. cit., p. 206.

${ }^{2}$ Immanuel Kant, Critique de la faculté de juger, $\S 5$.

${ }^{3}$ André Bazin, Qu'est-ce que le cinéma ?, op. cit., p. 312.

${ }^{4}$ Ibid., p. 280.

${ }^{5}$ Ibid., p. 307.
} 
donnée à voir comme poésie, «telle qu'en elle-même enfin, le cinéma la change $»^{1}$.

Et peut-être que la théorie du réalisme a toujours été avant tout cette redécouverte paradoxale du spectacle, des pures apparences, de la lueur, ce minimum de brillance nécessaire pour que le cinéma existe. Dans ce travail où est préservé le poids naturel des choses, le film laisse les êtres attirer l'attention sur eux suivant leur propre poids et se dédie à ce qu'ils puissent s'offrir à nous. En retour, les choses non manipulées se donnent «bénévolement » à cet art qui s'est ouvert à leur don. Quelle étrange circularité. Est-ce la tâche du cinéma, que d'être plus qu'un index, mais de veiller à la révélation du monde en imprimant cette veille sur la pellicule, à travers les empreintes lumineuses qui sont celles de la justice et de la justesse ? Pour Bazin, il semble que ce soit le cas : le dispositif machinique est apte à sauver l'honneur du réel, à déchiffrer et à révéler les apparences de la robe trop souvent déchiquetée de la réalité. La tonalité fondamentale de son œuvre, à la fois poétique, optimiste et rigoureuse, découle alors de l'idée que le cinéma est ce qui escorte avec pudeur la lumière du monde. Au croisement d'une technique qui destitue la présence de l'artiste, d'un mode de représentation presque candide et d'un régime de visibilité qui est celui de la faveur, où les libres apparences ne sont que la captation des ondes et des vibrations, d'événements de la présence matérielle, le cinématographe accomplit la promesse d'une reproduction mécanisée du monde capable de porter le poids de l'exigence pour l'artiste de cogner contre le réel afin de saisir «l'exacte concordance d'un surgissement éthique et d'un tracé esthétique $»^{2}$.

Le réalisme de Bazin, en ce sens, est moins ringard que tous ceux qui prétendent en finir avec lui. Dans les analyses pénétrantes de Qu'est-ce que le cinéma?, nous trouvons alors une eau qui était faite pour notre soif et qui n'a pas encore coulé.

${ }^{1}$ Ibid., p. 335 et p. 317.

${ }^{2}$ Jacques Rancière, La Fable cinématographique, op. cit., p. 197. 